\title{
Development of an engineering simulator for armored vehicle
}

\author{
Fang Tang \\ Wuhan Second Ship Design and Research Institute, 430000, Wuhan, China \\ tangfang008@163.com
}

Keywords: armored vehicle, engineering simulator, distributed interactive simulation, vehicle design, man-machine efficacy

\begin{abstract}
This paper illustrates the main features and structure of the armored vehicle engineering simulator (AVES). Considering high cost and long cycle development problem of the traditional armored vehicle design, the AVES has been developed for purposes to test rationality of design, to investigate different parameter effects on vehicle performance, to study man-machine efficacy in kinds of situation, to train crews. The AVES is made up of eight components, including vehicle dynamics, motion platform, visual simulation, sound effect, cockpit, man-machine interface, vibration seat and main console. High Level Architecture (HLA) pRTI and TCP/IP protocol is used to connect each component to exchange data and ensure real-time capability and synchronization of the AVES. Armored vehicle multi body dynamics model established by Vortex is reusable and modular to set up different vehicle. Motion platform is a classic Stewart platform driven by hydraulic system, which provides six degrees of freedom motion for crews. Visual simulation 3D model is built in Creator, rendered by VegaPrime to display on monitors in AVES. Man-machine interface and vibration seat is arranged in cockpit for crews operation. The development of the AVES can not only provide a platform for new vehicle design and performance evaluation, but also make further research on man-machine efficacy.
\end{abstract}

\section{Introduction}

Armored vehicle is a vital ground weapon, which has a very complicated mechanical structure and powerful function. In order to solve armored vehicle training problems of safety, economic, time, climate, field and other constraints, training simulator based on virtual simulation technology is applied. United States is one of the countries that firstly develops training simulator for armored vehicle and becomes leader of the world, M1AII tank training simulator is equipped to army in 2001, United States takes advantage of its computer network, uses LAN and WAN to connect other simulators around world, creates virtual battle environment and improves simulation reality[1]. Russian T72 tank driving simulator can be used for training by whole vehicle crew at the same time, easily to improve their group collaboration capability. European countries have also invested heavily in developing armored vehicle training simulators, including French "Leclerc" tank driving simulator, British "Challenger” tank driving simulator, German "Leopard 2" tank simulator[2]. China has also made some achievements in developing armored vehicle training simulator, JM3B system tank driving simulator[3][4] type 63A amphibious tank driving training simulator[5][6], the three crews-three degrees of freedom integrated training simulator developed by Guo Qisheng et.al[7] is based on distributed interactive simulation system which has cooperated training function.

Different from training simulator, armored vehicle engineer simulator (AVES) is a helpful tool for vehicle design, compared with traditional armored vehicle development mode "experience and experiment” which takes higher cost and longer cycle [8]. AVES not only provides a design and analysis platform in pre-research phase and program demonstration phase of new prototype vehicle which may speed up the development phase, but also tests rationality of design and gains useful information without risks of real vehicle experiment to improves vehicle performance and researches man-machine-environment relationships. Besides, it can be also used as training simulator for vehicle crew to improve their operating level. 
In this paper, key technique of developing the AVES is discussed. Section 2 gives an overview of the AVES, illustrates main components and network communication between them. Section 3 presents each component configuration, function and development technique in detail.

\section{Overview of armored vehicle engineering simulator}

The AVES is a typical man-machine-environment system, which studies about the relationships of man, machine and environment based on semi-physical simulation. The main components of the AVES are: vehicle dynamics, motion platform, visual simulation, sound effect, cockpit, man-machine interface, vibration seat and main console. The overall AVES architecture is shown in Fig.1. Main console sets all experiment parameters for the AVES, crews sit on vibration seat to operate inputs from man-machine interface that is the same as a real armored vehicle. These inputs are used to calculate vehicle state by vehicle dynamics model, the calculated result is send to motion platform to generate six degree of freedom motion, to vibration seat to simulate high frequency motion, to visual simulation to render realistic vehicle movement and visual picture, to sound effect to play different environment sound. Therefore, motion, view and sound works together to provide a better feeling for crews in cockpit to operate vehicle.

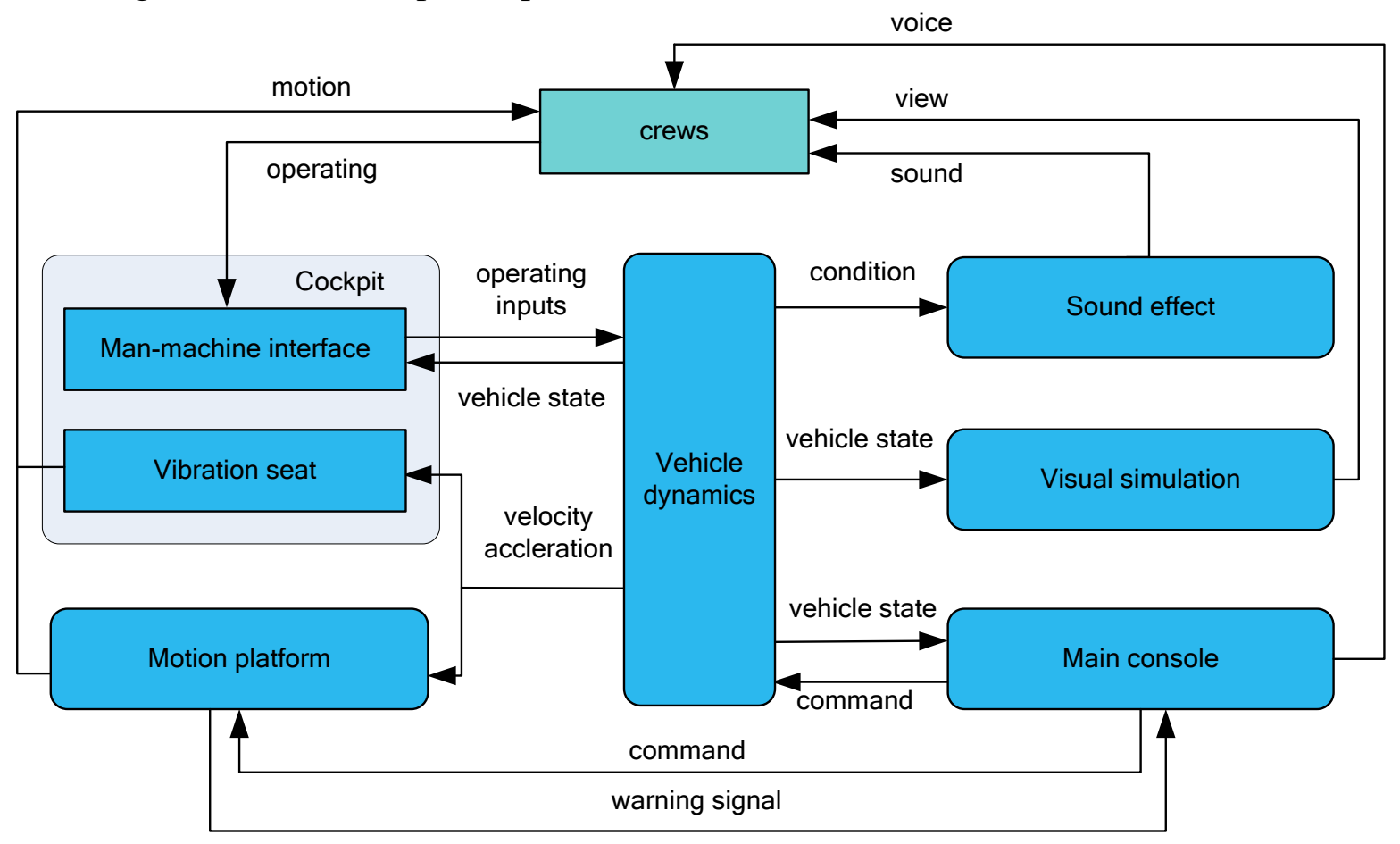

Figure 1. AVES architecture

The AVES is a complex distributed interactive simulation (DIS) system which involves more than ten computers. Three factors must be considered at least: 1) real-time capability; 2) synchronization; 3) expansibility. Considering above three factors, system network structure based on High Level Architecture (HLA) is used. HLA is a new DIS standard, compared with early DIS, HLA has less network data traffic, better reusability, flexibility and more powerful function[9].

In this network structure, vehicle dynamics is set as a core part communicating with others, HLA pRTI protocol is used to make data interaction with visual simulation, sound effect, man-machine interface, vibration seat and main console, coordinate synchronization of all above components; TCP/IP protocol is used to send data to the motion platform; and RS-232 serial communication is use to drive vibration seat.

\section{Development of armored vehicle engineering simulator}

Vehicle dynamics. The multi-body dynamics software Vortex is used to build this armored vehicle in AVES. In AVES simulation, barrel, turret, chassis, suspension and wheels are created as 
different parts, they are assembled together as a armored vehicle, then powertrain and control system is added to drive vehicle, material and contact property is set for terrain to build an accurate physical inactive simulation[10]. The rendering of Vortex armored vehicle simulation is shown in Fig.2. Meanwhile, developed by $\mathrm{C}++$ language, Vortex is easy to set up different vehicle models for AVES[11].

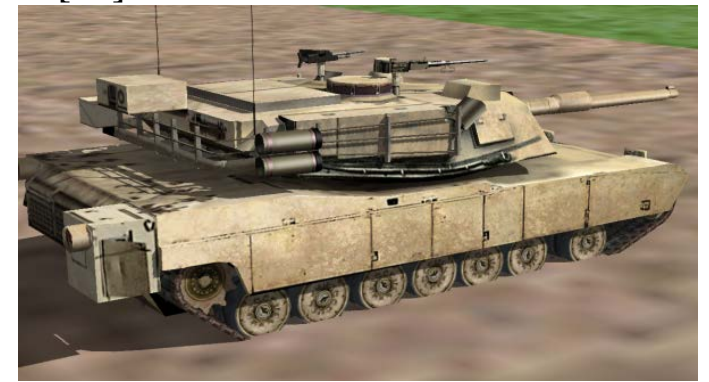

Figure 2. Rendering picture of Vortex

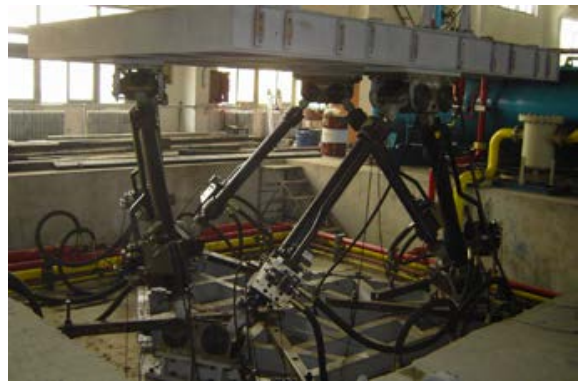

Figure 3. Motion platform

Motion platform. Motion platform is based on classic Stewart platform, it provides a realistic sense of vehicle movement for crew, simulates armored vehicle six directions movement: vertical, horizontal, vertical, pitching, rolling and heading. In AVES, motion platform is driven by hydraulic system, shown in Fig.3. The control system includes motion control computer, servo computer, oil monitoring computer, motion testing computer. Motion control computer receives acceleration and angular velocity data from vehicle dynamics computer using TCP/IP protocol, through Washout diagram, these data is send to servo computer, then servo computer controls hydraulic system to drive motion platform to simulate vehicle movement, and take back platform to balance position at appropriate time. Besides, motion control computer receives state parameters of motion platform send by servo computer, oil monitoring computer and motion testing computer using RS-232 serial communication. These parameters are used as a feedback signal for intelligent PID control.

Visual simulation. One significant factor for a good feeling of immersion in AVES is visual simulation. It contains graphic workstation, LCD monitor, video allocator, projector and projection screen. First of all, 3D models like vehicle, terrain database, natural landscape and humanity information are established by Creator. Then, visual image is rendered by graphic workstation using VegaPrime, including various grades of road, urban, rural, mountains, beaches and other terrain; sunny, cloudy, rainy, snowy, fog and other weather; day and night in different time; multi-channel scene rendering, shown in Fig.4. With video allocator, visual image is displayed in LCD monitors of cockpit and main console. The projector and projection screen is used to generate 180-degree virtual environment for crew looking outside.
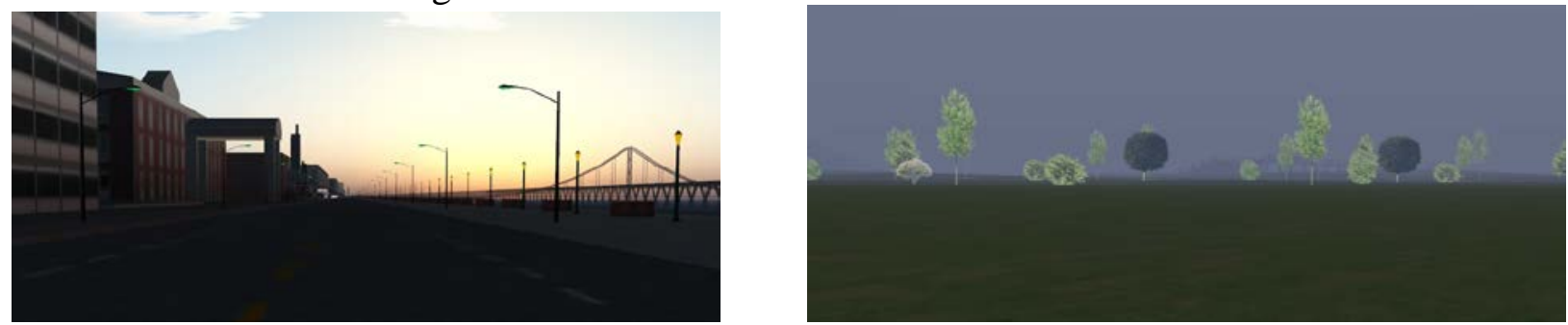

Figure 4. Visual simulation display image

Sound effect. The AVES can emulate sound that generate from engine, explosion, firing and so on. Different sound around crew's ear is recorded in real vehicle working condition like different engine sound in different shaft speed, optimal process is made using optimization software to form final sound database. In AVES simulation, according to vehicle working condition, computer selects appropriate sound $S$ from sound database:

$\mathrm{S}=\mathrm{f}(\mathrm{a}, \mathrm{b}, \mathrm{c}, \mathrm{d})$

where, a is gears, b is shaft speed, $c$ is ground condition, $d$ is other condition.

Sound S is played by OpenAL, which is built on DirectSound3D which is tightly integrated with VegaPrime.

Cockpit. AVES cockpit is installed in motion platform, and is the place that crews do simulation, so it is aimed to imitate real vehicle internal environment and function. The main parts of cockpit 
include man-machine interface and vibration seat.

Man-machine interface. It includes Driver operating force simulation and Other operating components. The typical components of driver operating force simulation are steering wheel, throttle pedal and brake pedal. The components can not only control vehicle, provide force feedback for driver in different working condition, but also acquire and record driver's operating data to analyze rationality of vehicle design which is an important part of man-machine engineering. Flexibility is also considered, components force feedback and operating displacement can be changed with different requirement. Steering wheel component contains steering wheel, torque sensor, angle sensor, torque motor, data acquisition card and industrial PC (IPC), value of force feedback is control by output voltage of IPC, its simulation process is shown in Fig.5.

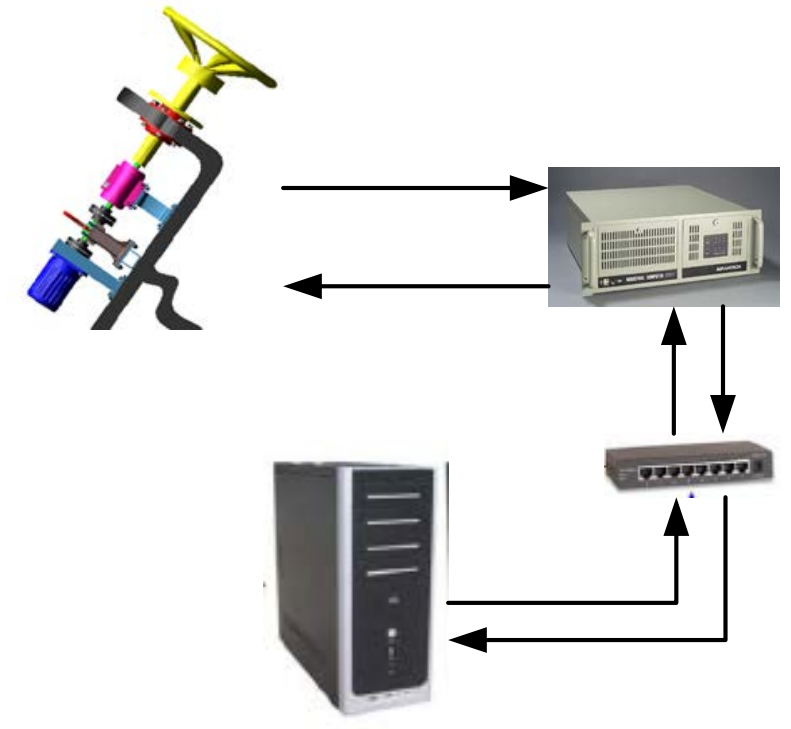

Figure 5. Steering wheel operating simulation

Other operating components include crew display control terminal, sight system and so on. They are reformed to receive and send data through network. For example, display panel receives vehicle state parameters from vehicle dynamics computer through HLA pRTI, and then updates its display.

Vibration seat. Engine shaft rotation, road roughness, firing and other reasons cause vibration when vehicle is running, motion platform can only provide low frequency vibration, and high frequency vibration is simulated by vibration seat to improve AVES motion fidelity.

Vibration seat provides vibration in vertical direction including two modes: composite mode and independent mode. In composite mode, high frequency vibration generated by vehicle velocity and acceleration is sent to vibration seat from motion platform using RS-232, motion platform works with vibration seat at the same time. While in independent mode, vibration seat directly simulates vertical vibration that is used to research psychological and physiological changes of crew in different frequency vibration.

Main console. Main console contains console software, control panel, video monitor and audio communicator. Console Software is developed with Visual Studio 2008, including setting condition module, control and run module, replay simulation module and analyze data module. In setting module, initial parameters such as virtual terrain, environment condition, vehicle parameters (e.g. mass, engine torque, engine speed and gear ratio) and material contact property (e.g. rolling resistance and slip ratio) is set. In control and run module, the AVES is ready to start a simulation, control command is sent and work state of each component is received by HLA pRTI, video monitor and audio communicator is used to observe crew's operation and communicate with them. While simulation, important data is saved (e.g. operating force, vehicle state) into SQL 2005 database in main console for analysis. In replay simulation module, crew can watch the replay that they have done to find out their mistake or weakness of operation and improve operation level. In analysis data module, according to database, vehicle state helps to optimize vehicle performance and design, components operating force and arrangement is analyzed from field of man-machine engineering to improve man-machine efficacy. 


\section{Conclusions}

In this paper, overview of the AVES is introduced, including main components and network communication between them, then each component configuration, function and development technique is described in detail.

AVES can be used in the whole process of vehicle design, which is low-cost and environmentally friendly compared with real vehicle experiment.

AVES vehicle dynamics is object oriented, therefore, it provides a flexible platform that is able to research the same vehicle type with different parameters.

Most parts inside AVES cockpit are flexible, which can help designer to improve man-machine efficacy. In the future, motion capture system can be added to AVES to record crew operating motion for data analysis, which leads further study of man-machine engineering.

AVES is able to set as a training simulator, its network structure based on HLA is reusable and easy to expand, which can be used to connect with other simulators to do large-scale simulation.

\section{References}

[1] Weir, D.H.,Ciark,A.J. A survey of Mid 2 Level Driving Simulators. SAE Paper. 1995, 01:72

[2] Ning Jia-kui. Research on visual simulaton system of tank driving and training simulator[D]. Jilin: Jilin University, 2007:2-4.

[3] Li Yu-ling, Zhang Sheng-xiang, Pan Wu-chao. Initiative infrared night viewing device design for JM series tank driving simulator[J]. Journal of System Simulation, 1995, S1:16-18.

[4] Wu Yao-wu, Xue Qing, Wang Jing-ye, et al. The CIG system of JM3B tank driving simulator[J]. Journal of System Simulation, 1995, S1:60-64.

[5] Zheng Chang-wei, Liu Yong-hong, Miao Zhuang, et al. Design and realization of scene simulation software for tank driving training simulator[J]. Computer Simulation, 1995, 22(11):263-266.

[6] Liu Yong-hong, Xue Qing, Guo Qi-sheng, et al. Posture simulation of the type 63A amphibious tank driving training simulator[J]. Computer Simulation, 2002, 19(3):15-17, 53.

[7] Guo Qi-sheng, Li Guang-hui, Zhang Gui-yuan, et al. Software system of the tank integrated training simulator[J]. Journal of System Simulation, 2001, 13(2):213-215.

[8] Han Bao-kun, Li Xiao-lei, Sun Feng-chun. Present state and future outlook of the simulation of tracked vehicles[J]. Acta Armamentrarii, 2003, 24(2): 246-249.

[9] CRAIN W, ZIMMERMAN P, TURRELL C. HLA technology transition: the challenge and the way forward[C]//Proceedings of the Fifth IEEE International Workshop on Distributed Simulation and Real-Time Applications. Cincinati, 2001:2-2.

[10] Tang Fang, Wei Yanding, Zhou Xiaojun, et al. Research on vehicle dynamics simulation for driving simulator. Advanced Materials Research, 2011, 308-310:1946-1950.

[11] CMLABS. Vortex document 4.1.2, 2009. 